See discussions, stats, and author profiles for this publication at: https://www.researchgate.net/publication/4323306

\title{
Performance improvement of wireless network based on effective data transmission
}

Conference Paper · February 2008

DOI: 10.1049/cp:20080163 · Source: IEEE Xplore

CITATIONS

5

3 authors, including:

Samuel Ndueso John

Nigerian Defence Academy

48 PUBLICATIONS 170 CITATIONS

SEE PROFILE
READS

302

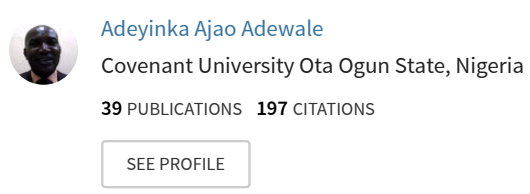

Some of the authors of this publication are also working on these related projects:

Project Improving Prioritized Handover Performance for Mobile WiMAX by Dynamic Guard Channel Allocation and RSS Quality Factor View project

Software Defined and Cognitive Radio Testbed (SoDCRaT) View project 


\title{
Performance Improvement of Wireless Network Based on Effective Data Transmission
}

\author{
*S.N. John, F.A. Ibikunle, A.A. Adewale \\ *Covenant University, Ota, Ogun State, Nigeria. \\ johnsam8@hotmail.com
}

Keywords: Transmission, Bandwidth, Networks, Workload.

\begin{abstract}
A major requirement of any computer network is scalability. This can be as a result of increase in the number of users, upgrading due to technological advancement, additional services, or the need for performance improvement. This will inevitably require changing infrastructure, deploying new applications, and dealing with security which definitely touches both hardware and software, in the sense that, the complexities of the hardware, software and firmware increase with the attendant growth of the network and maintainability. However, for the network to remain reliable and efficient, it is necessary that, implementation and administration of it requires a means of monitoring both the model of the network structures and processes occurring in them. The efficiency and throughput of the network depend on improving the effectiveness of data transmission carried out by the network protocols. This paper discusses the use of a protocol to meet the technological challenge towards improving the performance and throughput of a wireless network based on effective data transmission.
\end{abstract}

\section{Introduction}

A computer communication network is never appreciated a major asset until when there is performance degradation - a situation when the network starts crawling at peak period thereby creating problems for local hosts and also, when remote users find it a difficult task to connect to the network. In such cases frustration sets in especially when downtime becomes prolonged, let alone lost in revenue, profitability, bandwidth consumption and typing up of IT resources until these problems are resolved the huge cost on investment will not be justified.

However, contingency planning is very crucial in averting network breakdown or network traffic downtime as described above and one way achieving a good quality network carrying out a network modeling and simulation. Simulation has become a necessary tool in studying network. The use of simulation programs on TCP/IP protocol with special attention on mode of data transmission will provide an improvement in bandwidth utilization even at peak periods.

Different researchers have looked into the network optimization issue and carried out work on improvement of bandwidth usage. Polly Huang and John Heidemann discovered TCP burstiness in light-weight simulations of data by counting packets with inter arrival times falling into certain critical intervals - round trip time (RTT) or retransmission timeout (RTO), they were able to create finite state automaton (FSA) with states and transitions indicating rounds of back-to-back packet transmissions. They demonstrated that an FSA approximation of TCP can produce lightweight simulation models of TCP suitable for background traffic, and that these models accurately reproduce multi-fractal scaling behavior in IP network traffic [1]. Increasing the effectiveness of data exchange within a network based on the protocol of TCP/IP using simulation modeling were looked into and the result obtained showed that it is possible to maximize bandwidth efficiency by the mode of data transmission[2].

The key contribution of this paper is to develop a new approach of optimizing data transmission rate that leads to improvement in bandwidth utilization.

This paper is organized as follows: Section 2 reviews the problems associated with wireless networks while Section 3 deals with the Influence of data rates on transmission efficiency of wireless networks. In Section 4, the graphical results obtained from simulation were analyzed and inferences drawn.

\section{Problems associated with wireless networks}

Lately, with emergence of new technologies, there is continual growth and increase in complication of network projects. Every year, the intense growth of user and scalability of different corporate network, including the university networks has push the computer network beyond what it used to be - an experimental network. Meanwhile, it is necessary to use special means in analyzing and designing network connection because of the increasing complications of network infrastructure.

Wireless networks encounter problems like various forms of delay or latency, topology, hardware, excessive bandwidth consumption and mode of data exchange culminate in network traffic congestion and eventually packet loss. Also, the way data are managed during transmission by the protocols handling the data transfer can really reduce the performance of a wireless and fixed local area network. The main task of the analysis and modeling of the modes of data exchange in modern computer networks based on TCP/IP protocol is for increasing the performance efficiency of the 
network and network application, and above all, increasing their productivity.

Thus, the main subject of investigation becomes mode of data exchange towards increasing the performance of wireless computer networks.

\section{Influence of mode of data transmission on the efficiency of wireless networks.}

Considering that the workload on the network are uniformly distributed among the nodes, as shown in Fig. 1 below, then with the aid of computer programs, the analysis of traffic passing through all the nodes in the network can be done and statistical information obtained and preliminary analysis of the workload on the communication channels can be carried out.

The bandwidth capacities of a communication channel is determined by the set of network infrastructure, desired workload - network structure (number of computers, topology of their connection), also users and applications.

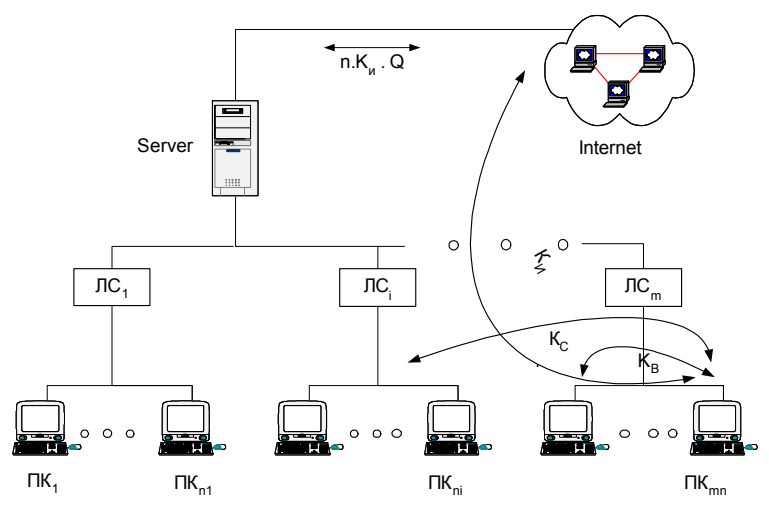

Fig. 1. Analytical scheme of corporate network

The desired workload can be determined analytically taking into account the following assumptions:

- average quantity of transfer information per time unit on one computer been equal to all computers on the network $\left(Q_{1}\right)$;

- specific gravity of transfer information on the Internet generally the volume of data transfer is approximately identical for all computers as compared to internal network and determined by the coefficient $\left(\mathrm{K}_{\mathrm{N}}\right)$;

- at the same time the activities on the networks are done with some restrictions

- $\quad$ specific gravity of transfer information on a network is characterized by the coefficients $K_{B}-$ the probability of sending/receiving of data for computers from other subnets/networks and $\mathrm{K}_{\mathrm{C}}-$ the probability of sending/receiving of data for computers on the same subnet.

Obviously, this gives the following equations:

$$
\mathrm{K}_{\mathrm{N}}+\mathrm{K}_{\mathrm{B}}+\mathrm{K}_{\mathrm{C}}=1
$$

4Work load on link Channel - coefficient of work load $\left(K_{H}\right)$ :

$$
K_{H}=\frac{Q_{L C}}{Q_{n}},
$$

Where $Q_{L C}$ - Desired workload on link channel

$$
Q_{n} \text { - Normal bandwidth on link channel }
$$

Work load of data transfer on the internal link:

$$
Q_{N}=Q_{1} \bullet K_{N} \bullet n, \quad n=\sum_{i=1}^{m} N_{i}
$$

Where $n$ - number of computers on the LAN

$Q_{1}$ - average volume of data exchange for a node in a unit time

$K_{N}$ - probability of exchange of information on Internet

$N_{i}$ - number of computers on $\mathrm{i}=$ subnet (including those connected to the subnet of lower layer)

$\mathrm{m}$ - number of subnet on the LAN

Work load of data exchange on the bus of subnet LAN:

$$
Q_{L C i}=Q_{1} \bullet N_{i}
$$

Work load of data exchange on LAN tunnel:

$$
Q=Q_{1}\left[K_{u} \cdot n+\frac{1-K_{N}}{n-1} \sum_{I=1}^{m} N_{i}\left(n-N_{i}\right)\right]
$$

The use of specialized means in analyzing, modeling and researching of packet exchange in large-scale corporate computer network (for example university network infrastructure), extreme complexity of corporate intranet networks and the Internet make the development of analytic models very difficult and under such circumstances; simulation models are viable alternatives that help in understanding the behavior of these networks during data exchange in a corporate intranet such as figure 1. In this case, the primary research of data exchange in a network using analytical dependences that allows approximate estimate of the workload of two layers of corporate network as shown in figure 1. However, the real corporate networks of major concerns and the universities as an example have greater number of levels, which substantially hampers the use of calculation/analytical methods and requires application of multilevel imitation design of network infrastructure. In realizing this, a specialized simulation technique such as NetCracker professional was used. Hence, a research on workload capacity of an external network was carried out using the following parameters:

- size of the send files,

- number of connections,

- time domain between the transmissions of the files. 
Developed in the process of simulation is a model containing 4 layers/levels that consists of 11 models campuses/buildings of 1100 computers on the network.

\section{Results of simulation}

Table 1 shows the results obtained from simulation which is depicted graphically in figure 2. Here, it is observed that increasing the file size sent (i.e., at a fixed parameter of data flow) and the channel workload will results in increase in the effective bandwidth capacity toward maximum level (ranging from 128 to 1024 Kbytes for different networks). This will on the other hand, affect the overall bandwidth capacity and results in the reduction of the total bandwidth.

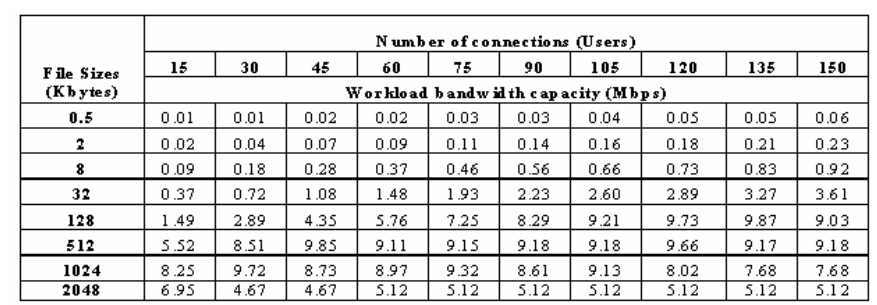

Table 1: Results of Simulation

The achieved dependants allow defining, how concretely an increase in send file sizes affects the effectiveness of bandwidth capacity on a network.

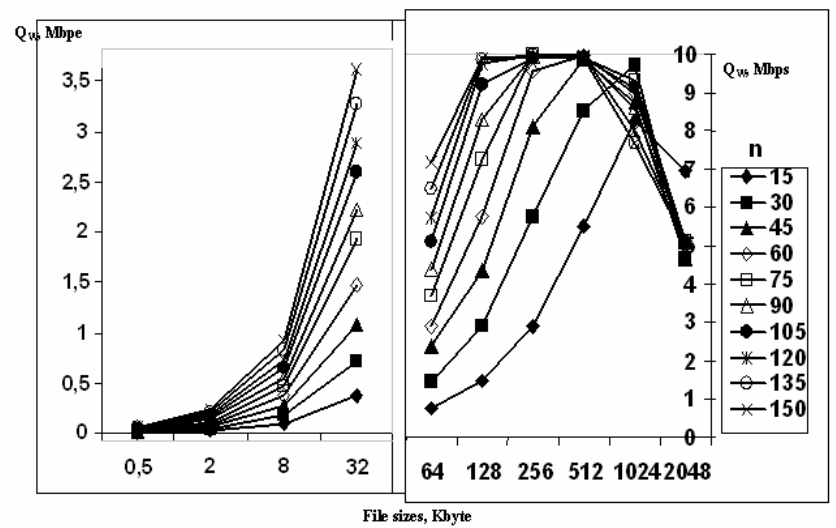

Fig. 2: The graph shows the bandwidth capacity of external channel on a corporate network based on an average file size and different number of network connections

Table 2 shows the dependant bandwidth capacity of external network using an average file sizes from different number of network connections as shown graphically in figure 3 .

From the graph it is seen that a change in file size sent from 128 - 512 Kbytes have no substantial influence on the bandwidth capacity of the external network both at the intial workload of the network ( 15 connections, $\left.\mathrm{Q}_{\mathrm{TN}}=0,61\right)$, and at the workload (30 -150 connections, $\left.\mathrm{Q}_{\mathrm{TN}}=15\right)$.

\begin{tabular}{|c|c|c|c|c|c|c|c|c|c|c|c|}
\hline \multirow{3}{*}{$\begin{array}{c}\text { File } \\
\text { Size, } \\
\text { (Kbytes) }\end{array}$} & \multicolumn{10}{|c|}{ Num ber of connection s (U ser s) } \\
\cline { 2 - 10 } & $\mathbf{1 5}$ & $\mathbf{3 0}$ & $\mathbf{4 5}$ & $\mathbf{6 0}$ & $\mathbf{7 5}$ & $\mathbf{9 0}$ & $\mathbf{1 0 5}$ & $\mathbf{1 2 0}$ & $\mathbf{1 3 5}$ & $\mathbf{1 5 0}$ \\
\hline $\mathbf{0 , 5}$ & 6,00 & 9,71 & 9,84 & 9,84 & 9,84 & 9,84 & 9,84 & 9,84 & 9,84 & 9,84 \\
\hline $\mathbf{2}$ & 6,01 & 9,48 & 9,48 & 9,48 & 9,48 & 9,48 & 9,48 & 9,48 & 9,48 & 9,48 \\
\hline $\mathbf{8}$ & 5,97 & 9,48 & 9,48 & 9,48 & 9,48 & 9,48 & 9,48 & 9,48 & 9,48 & 9,48 \\
\hline $\mathbf{3 2}$ & 5,99 & 9,84 & 9,71 & 9,78 & 9,71 & 9,65 & 9,65 & 9,65 & 9,97 & 9,78 \\
\hline $\mathbf{1 2 8}$ & 6,02 & 9,79 & 9,84 & 9,84 & 9,84 & 9,84 & 9,84 & 9,84 & 9,84 & 9,84 \\
\hline $\mathbf{5 1 2}$ & 5,98 & 8,76 & 9,87 & 9,24 & 9,71 & 9,65 & 9,60 & 9,71 & 9,65 & 9,65 \\
\hline $\mathbf{1 0 2 4}$ & 4,91 & 7,67 & 8,82 & 8,45 & 9,29 & 9,13 & 9,34 & 9,32 & 9,05 & 8,90 \\
\hline $\mathbf{2 0 4 8}$ & 3,71 & 6,08 & 7,04 & 7,36 & 7,43 & 6,80 & 7,26 & 6,66 & 7,23 & 6,85 \\
\hline
\end{tabular}

Table 2: Results of dependant bandwidth capacity
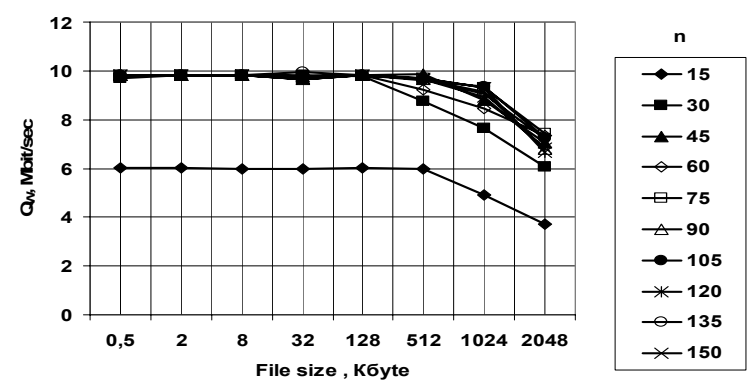

Fig. 3: The graph of bandwidth capacity of an external channel on a corporate network based on average file size, different number of network connections and workload capacity.

Further increase of workload to the network, makes the bandwidth capacity of the network to decrease (in this case at an average send file size of not more than 512 Kbytes). Thus, for a corporate network at a given condition of the maximum send file size exceeding of which results in the substantial decline in the efficiency of data exchange.

For the purpose of verification and authentication of the obtained results, the real model experiment was conducted. The effect of file size transfer from eternal network to the server and also server to the external workstation were compared with the modeled experiment.

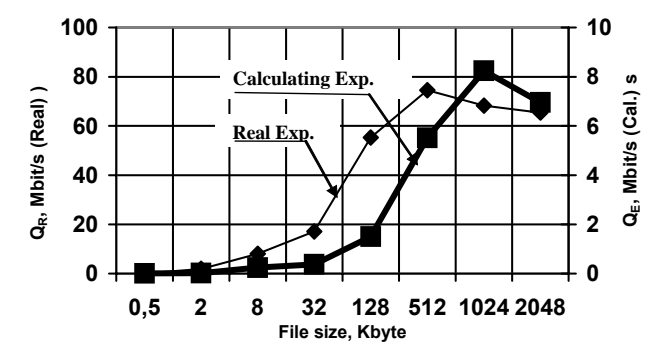

Fig. 4. Research result of workload from main computing network system obtained by model and the Computer

During the processes of transmission, the handshake time and average bandwidth performance were noted. The result obtained from the modeled experiment was compared with the results of analytical experiment as shown in figure 4 . 
Which depicts the Graph of $\mathrm{Q}_{\mathrm{R}}(\mathrm{L})$ obtained from computing experiment from 15 connections and period of transmission of files, evenly distributed with an interval 1-19 seconds. The analysis of dependences confirms in conclusion the existence of maximum file size, of 512 Kbytes obtained experimentally. It describes the method of increase the efficiency of network data transmission based on using the developed models and dependence results. Also, is the joint developed methodology of different means of designing and analyzing the network infrastructure with the aim of increasing the efficiency of packet transmission based on the TCP/IP protocols stack.

The summary results shows the possibility to conclude that, the mode of data exchange can substantially have influence on the efficiency and performance of the TCP/IP network as shown in the figure 4. As a whole, summarizing the results of the researches [2], the three-dimensional graph represented in Fig. 4, shows how the mode of data exchange depends strongly on the bandwidth capacity of a network. The results were obtained by the use of imitational simulation of network nodes using Matlab and Netcracker simulation programs, and this shows the level in which mode of data transmission affects the network performance.

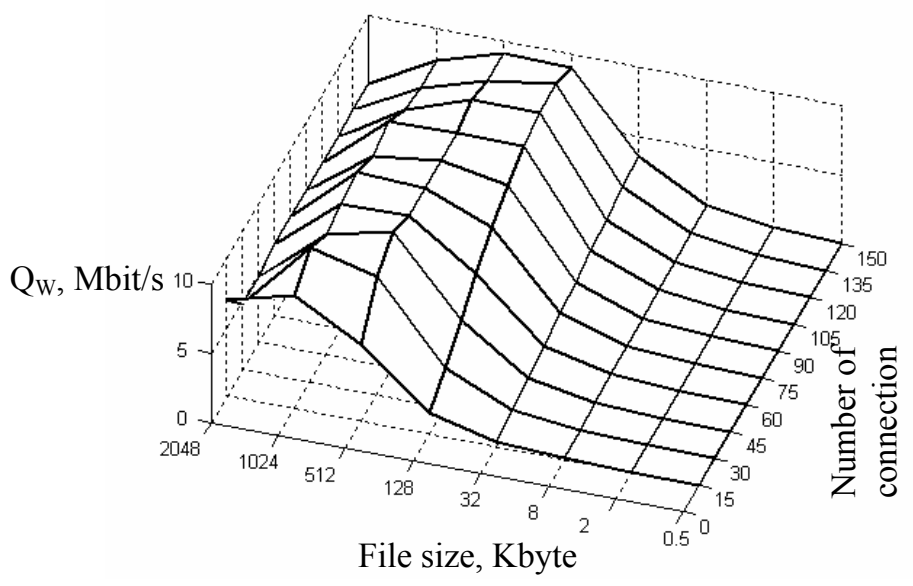

Fig. 4. Dependant bandwidth capacity of a network at number of connections and send file size

Thus, the achieved result gives an opportunity to offer a special method in increase the efficiency of data exchange in a distributed computer network. Essence of the offered method consists in the sharing of the dependences got from both the analytical and experimental way, and also complex developing simulation models, for deciding the most effective mode of data exchange during a given condition on exploitation of computer network and network applications. For realization the given method the following execution sequence is offered:

1. Preliminary determine the character of different modes of data exchange in an explore network environment through developed models of physical and transport levels or the achieved dependences got from the research work.

2. Determine possible variants of network structure or network application.
3. Investigating the different variants in realizing the critical productivity distributed computer networks using the simulation technique system of NetCracker.

4. Clarification where necessary a separate characteristic of the modes of data exchange in a critical through productivity fragments of an explored network environment.

5. Making recommendations on the most rational organization of data exchange in a computing network within the framework on the explored network application.

Method of increasing the effectiveness of network data exchange based on using the imitation models and all the basic data, imitation modeling, the dependences with the analytical estimation and some crucial recommendations. By applying the steps given we can successful improve the performance of WLAN based on the data exchange management.

\section{Conclusion}

In this paper, we presented a model for generating and realizing workload distributions for corporate intranet network and Internet using the Matlab/Simulink and NetCracker applications. This model results captures the temporal and spatial interactions between the sources and with their network connections. The analytical method has a limit due to network complication, and the simulation method gives the optimum results. Sustainable development in IT needs this research work to influence their network performance and efficiency. Based on the results of imitation simulation (for example corporate network) with the use of the NetCracker Professional system, the maximum parameters values of workload which if made to exceed the file size (e.g., 512 Kbytes) will results in substantial declination of the efficiency and the bandwidth capacity of computer networks.

\section{References}

1. P. Huang and J. Heidemann. Capturing TCP Burstiness for Lightweight Simulation. Technical Report TIK-Nr.92, Computer Engineering and Networks Laboratory, Swiss Federal Institute of Technology, Zurich, 2000.

2. S. N. John: "Increasing the Efficiency of Data Exchange in a Computer Networks based on the Protocol of TCP/IP Suite", Scientific Journals: Information, cybernetics and Computing Engineering: Donetsk ( DonNTU), Ukraine, Vol. 93, pp. 256-264, 2005.

3. Olifer V.G., OLifer N.A. Computer network. Principles of technologies, protocols - SPB: Publication «Inter», 1999. 668 p.: illustration.

4. Stevens W.R. TCP slow start, congestion avoidance, fast retransmit, and fast recovery algorithms. RFC 2001, Internet Request For Comments, January 1997.

5. Stevens W.R. TCP/IP Illustrated, Volume1: The Protocols, 1998, 576p. 